\title{
Why disulfiram is not a panacea?
}

\author{
Boris Cvek ${ }^{1}$ \\ ${ }^{1}$ Palacky University Olomouc
}

October 20, 2020

\begin{abstract}
Two recent studies in Nature and Nature Immunology suggest that an old anti-alcoholism drug disulfiram could treat covid19 and sepsis, respectively [1-2]. In fact, disulfiram is highly reactive compound (creating both S-S bonds with thiols and chelates with metals) which almost does not exist in the body early after ingestion (the anti-alcoholism effect is caused by its metabolites). [3]
\end{abstract}

Why disulfiram is not a panacea?

Two recent studies in Nature and Nature Immunology suggest that an old anti-alcoholism drug disulfiram could treat covid-19 and sepsis, respectively [1-2]. In fact, disulfiram is highly reactive compound (creating both S-S bonds with thiols and chelates with metals) which almost does not exist in the body early after ingestion (the anti-alcoholism effect is caused by its metabolites). [3]

Under in vitro conditions, disulfiram is able to react with tens of proteins pretending that it is a panacea (indeed, many other studies have found simply on the basis of in vitro data that disulfiram targets distinct proteins in models of various diseases). It is not. Meaningful biomedical research focused on disulfiram should identify the active compound, i.e. a disulfiram metabolite, in vivo and demonstrate coherence of both in vitro (the experiments with the disulfiram metabolite) and in vivo data.

Boris Cvek (cvekb@seznam.cz)

Olomouc University Social Health Instituce (OUSHI), Czech Republic

ORCID: http://orcid.org/0000-0001-8238-3890

Citations:

[1] Jin, Z. et al. (2020) Structure of $\mathrm{M}^{\text {pro }}$ from COVID-19 virus and discovery of its inhibitors. Nature 582, 289-293.

[2] Hu, J.J. et al. (2020) FDA-approved disulfiram inhibits pyroptosis by blocking gasdermin D pore formation. Nat. Immunol. 21, 736-745.

[3] Eneanya D.I. et al. (1981) The actions and metabolic fate of disulfiram. Ann. Rev. Pharmacol Toxicol. $21,575-596$ 